Canad. Math. Bull. Vol. 22 (3), 1979

\title{
BOUNDS ON POSITIVE INTEGRAL SOLUTIONS OF LINEAR DIOPHANTINE EQUATIONS II
}

BY

\author{
I. BOROSH* AND L. B. TREYBIG
}

\begin{abstract}
Let $A$ be an $m \times n$ matrix of rank $r$ and $B$ an $m \times 1$ matrix, both with integer entries. Let $M_{2}$ be the maximum of the absolute values of the $r \times r$ minors of the augmented matrix $(A \mid B)$. Suppose that the system $A x=B$ has a non-trivial solution in nonnegative integers. We prove (1) If $r=n-1$ then the system $A x=B$ has a non-negative non-trivial solution with entries bounded by $M_{2}$. (2) If $A$ has a $r \times n$ submatrix such that none of its $r \times r$ minors is 0 and $x \geq 0$ is a solution of $A x=B$ in integers such that $\sum_{i=1}^{n} x_{i}$ is minimal, then $\sum_{i=1}^{n} x_{i} \leq\left(n r+n-r^{2}\right) \mathrm{M}_{2}$.
\end{abstract}

Introduction. In [1] the following problem was considered: Let $A$ be an $m \times n$ matrix, $B$ an $m \times 1$ matrix, both with integral entries, and consider the system of equations:

$$
A x=B \text {. }
$$

Suppose (1) has a non-zero solution in non-negative integers. The problem is to find a bound $K=K(A, B)$ such that the existence of such a solution with entries bounded by $K$ is always guaranteed. The problem first arose in a topological setting $[3,4,5]$ and a bounding function $K=K(A, B)$ was found inductively in [5]. Let $(A \mid B)$ denote the augmented matrix of $(1) ; r$ denote the rank of $A ; M_{1}, M_{2}$ denote, respectively, the maximum of the absolute values of all the minors of order $r$ of $A$ and $(A \mid B)$; and $M$ the maximum of the absolute values of all the minors of $(A \mid B)$.

It was conjectured in [1] that if (1) has a non-trivial solution in non-negative integers, then it has one whose entries are bounded by $\mathbf{M}_{2}$. The above bound is clearly sharp. The conjecture was proved in [1] only for the case $m=1$ and the case in which the homogeneous system $A x=0$ has no non-trivial non-negative solutions. In [2] the conjecture was proved for the homogeneous case. Also in [1], the bound $M_{2}\left(1+1 / M_{1}\right)$ was obtained for the case $r=n-1$ and a bound of the order of $\mathrm{M}^{2}$ was obtained in the general case.

In this paper the conjecture will be proved for the case $r=n-1$, and a better bound of the order of $\mathrm{M}_{2}$ will be obtained in the general case. The main results are:

THEOREM 1. If $r=n-1$ and (1) has a non-trivial non-negative solution then it has such a solution with $\max _{i} x_{i} \leq \mathrm{M}_{2}$.

Received by the editors August 3, 1977 and in revised form, September 12, 1978.

* This author was partially supported by NSF grant MCS 76-06092. 
THEOREM 2. If none of the minors of order $r$ of $A$ is 0 and $x=\left(x_{1}, \ldots x_{n}\right)$ is a non-trivial non-negative solution in integers of (1) such that $\sum_{i=1}^{n} x_{i}$ is minimum, then $\sum_{i=1}^{n} x_{i} \leq\left(n r+n-r^{2}\right) \mathrm{M}_{2}$.

Proof of Theorem 1. We may suppose without loss of generality that $m=r$ and that $\max x_{i}$ is a minimum over all such solutions. Also, assume that the variables have been renamed so that $x_{1} \leq x_{2} \leq \cdots \leq x_{r+1}$ and that $\mathrm{M}_{2}<x_{r+1}$. We multiply both sides of $A x=B$ by the adjoint of the matrix $A^{\prime}$ whose columns are the first $r$ columns of $A$, and easily derive:

$$
c x_{i}=-n_{i}+p_{i} x_{r+1}, \quad 1 \leq i \leq r,
$$

where each $-n_{i}, p_{i}, c$ or its negative is the determinant of an $r \times r$ submatrix of $(A \mid B)$. We may assume without loss of generality that $c \geq 0$.

If $c=0$, then $-n_{i}+p_{i} x_{r+1}=0,1 \leq i \leq r$. But $p_{i}=0,1 \leq i \leq r$, implies the rows of $A$ are not linearly independent, and some $p_{i} \neq 0$ implies $x_{r+1} \leq\left|n_{i}\right|$. Thus $c>0$. Also notice that if $x_{i}=x_{r+1}$ for $i \leq r$, then $p_{i} \neq 0$ since $p_{i}=0$ would imply $x_{i} \leq \mathrm{M}_{2}<x_{r+1}$.

If $p_{i}<0$ for some $i$, then (2) implies $c x_{i}-p_{i} x_{r+1}=-n_{i}$, which is impossible since $x_{r+1}>\left|n_{i}\right|$. Thus $p_{i} \geq 0,1 \leq i \leq r$. If $p_{i} \leq x_{i}, 1 \leq i \leq r$, then $\left(x_{1}-p_{1}, \ldots\right.$, $\left.x_{r}-p_{r}, x_{r+1}-c\right)$ is a non-trivial solution with a smaller maximum, a contradiction. Suppose then that $x_{i}<p_{i}$.

Now, $x_{r+1}>c, x_{i}<p_{i}$, and (2) imply:

$$
n_{i}=x_{r+1} p_{i}-x_{i} c=x_{i}\left(x_{r+1}-c\right)+\left(p_{i}-x_{i}\right) x_{r+1} \geq x_{r+1},
$$

a contradiction.

COROLlaRY. If $r=n-1$ and (1) has a solution in integers, then it has a solution in integers such that $\max _{i}\left|x_{i}\right| \leq \mathrm{M}_{2}$.

Proof. Suppose that (1) has a solution in integers $x_{1}, \ldots x_{n}$ and assume $x_{1}, \ldots, x_{k} \geq 0$ and $x_{k+1}, \ldots, x_{n}<0$. Define

$$
y_{i}=\left\{\begin{array}{rr}
x_{i} & i \leq k \\
-x_{i} & i>k
\end{array}\right.
$$

Let $\bar{A}$ be the matrix obtained from $A$ by changing the signs of the columns $k+1, \ldots, n$ of $A$. Then, the system $\bar{A} x=B$ has the non-trivial solution $y$ and, therefore, by Theorem 1 , has a solution bounded by $M_{2}$ (since the absolute value of the minors of $A$ and $\bar{A}$ are equal). A solution to (1) is then easily obtained by adjusting the signs.

Proof of Theorem 2. We may assume without loss of generality that $r=m$ and that $x_{1} \geq x_{2} \geq \cdots \geq x_{n}$. For $i=0,1, \ldots r$, let $S_{i}$ denote the set of all $r \times r$ 
submatrices of $A$ whose first $i$ columns coincide with the first $i$ columns of $A$. In particular, $S_{0}$ is the set of all $r \times r$ submatrices of $A$. In particular, $S_{0}$ is the set of all $r \times r$ submatrices of $A$. Let $D_{i}$ denote the maximum of the absolute values of the determinants of $S_{i}$ for $i=0, \ldots, r$. We have obviously: $\mathrm{M}_{1}=D_{0} \geq$ $D_{1} \geq \cdots \geq D_{r}$. We now distinguish between two cases:

CASE 1. For every $j, j=0, \ldots, r-1$, we have

$$
x_{j+1} \geq D_{j} \text {. }
$$

Let $D=D_{r} \neq 0$, and assume, without loss of generality that $D>0$. Let $A^{\prime}$ be the submatrix whose columns are the first $r$ columns of $A$. Solving for $x_{1}, \ldots, x_{r}$ we get:

$$
D x_{i}=\sum_{k=r+1}^{n} a_{i k}^{\prime} x_{k}+b_{i}^{\prime}
$$

where $a_{i k}^{\prime}$ is the determinant of the $r \times r$ submatrix of $A$ obtained from $A^{\prime}$ by replacing the $i$ th column of $A$ by $k$ th column. This submatrix belongs to $S_{i-1}$ and therefore $\left|a_{i k}^{\prime}\right| \leq D_{i-1}$. The term $b_{i}^{\prime}$ is the minor obtained by replacing the $i$ th column of $A^{\prime}$ by $B$. Let $v$ be the largest integer $j$ where $x_{j-1} \geq D$. From (3) we see that $v \geq r+1$. If $v>r+1$ let $p$ be any integer such that $r+1 \leq p<v$, and let $m_{p}=\sum_{i=1}^{r} a_{i p}^{\prime}+D$. If $m_{p} \neq 0$, define a new solution $y$ of (1) as follows:

$$
y_{j}= \begin{cases}x_{j} \text { if } \quad j \geq r+1, & j \neq p \\ x_{p}-\left(\operatorname{sgn} m_{p}\right) D & j=p \\ x_{j}-\left(\operatorname{sgn} m_{p}\right) a_{j p}^{\prime} \text { for } & j=1, \ldots, r\end{cases}
$$

In (5) $\operatorname{sgn} m_{p}=+1$ if $m_{p}>0$ and -1 if $m_{p}<0$. It is easily seen from (4) that $y$ is a solution to (1). Since $p<v, x_{p} \geq D$. So $y_{p}=x_{p}-\operatorname{sgn} m_{p} D \geq 0$. Since $\left|a_{i p}^{\prime}\right| \leq$ $D_{j-1}$ and from (3), $x_{j} \geq D_{j-1}$ we have for $j=1, \ldots, r y_{j}=x_{j}-\left(\operatorname{sgn} m_{p}\right) a_{j p}^{\prime} \geq 0$.

$$
\begin{aligned}
\sum_{j=1}^{n} y_{j} & =\sum_{j=1}^{n} x_{j}-\operatorname{sgn} m_{p}\left(\sum_{j=1}^{r} a_{j p}^{\prime}+D\right)=\sum_{j=1}^{n} x_{j}-\left|m_{p}\right| \\
& <\sum_{j=1}^{n} x_{j} .
\end{aligned}
$$

This contradicts the minimality of $\sum_{1}^{n} x_{j}$. We have therefore $m_{p}=0$ and:

$$
\sum_{i=1}^{r} a_{i p}^{\prime}=-D, \quad p=r+1, \ldots, v-1
$$

Summing (4) for $i=1, \ldots, r$ we get:

$$
D \sum_{i=1}^{r} x_{i}=\sum_{p=r+1}^{v-1}\left(\sum_{i=1}^{r} a_{i p}^{\prime}\right) x_{p}+\sum_{p=v}^{n}\left(\sum_{i=1}^{r} a_{i p}^{\prime}\right) x_{p}+\sum_{i=1}^{r} b_{i}^{\prime} .
$$


Using (6) we get:

$$
D \sum_{i=1}^{v-1} x_{i}=\sum_{p=v}^{n}\left(\sum_{i=1}^{r} a_{i p}^{\prime}\right) x_{p}+\sum_{i=1}^{r} b_{i}^{\prime}
$$

and since $x_{p}<D$ for $p \geq v$;

$$
\begin{aligned}
\sum_{i=1}^{v-1} x_{i} & \leq(n-v+1) r \mathrm{M}_{1}+r \mathrm{M}_{2} \\
\sum_{i=1}^{n} x_{i} & \leq((n-v+1) r+(n-v+1)) \mathrm{M}_{1}+r \mathrm{M}_{2} \\
& \leq(n-v+1)(r+1) \mathrm{M}_{1}+r \mathrm{M}_{2} \\
& \leq(n-r)(r+1) \mathrm{M}_{1}+r \mathrm{M}_{2} \\
& \leq\left(n r+n-r^{2}\right) \mathrm{M}_{2} .
\end{aligned}
$$

If $v=r+1$, then the first term on the right side of (7) may be replaced by zero.

CASE 2. There exists $j, 0 \leq j<r$ such that $x_{j+1}<D_{j}$. We rename the variables $x_{j+1}, \ldots, x_{n}$ in such a way that the matrix $A^{\prime}$ whose columns are the first $r$-columns, has determinant $D= \pm D_{j}$; and we may assume $D=D_{j}$. We solve for $x_{1}, \ldots, x_{r}$ :

$$
D x_{i}=\sum_{p=r+1}^{n} a_{i p}^{\prime} x_{p}+b_{i}^{\prime}
$$

where $a_{i p}^{\prime}$ and $b_{i}^{\prime}$ are minors of $A$ and $(A / B)$ as in Case 1. Since $x_{p}<D$ for $p=r+1, \ldots, n$ we have $x_{i} \leq(n-r) \mathrm{M}_{1}+\mathrm{M}_{2}, i=1, \ldots, j$

$$
\begin{aligned}
\sum_{i=1}^{n} x_{i} & \leq j(n-r) \mathrm{M}_{1}+(n-j) \mathrm{M}_{1}+j \mathrm{M}_{2} \\
& \leq(j(n-r-1)+n) \mathrm{M}_{1}+j \mathrm{M}_{2} \\
& \leq(r+1)(n-r) \mathrm{M}_{1}+r \mathrm{M}_{2} \\
& \leq\left(n r+n-r^{2}\right) \mathrm{M}_{2} .
\end{aligned}
$$

REMARK. In the proofs of Theorems 1 and 2 we assume that $r=m$. In fact, we can choose among the $r$-tuples of rows of $(A \mid B)$ the one for which $M_{2} \neq 0$ is minimal.

\section{REFERENCES}

1. I. Borosh and L. B. Treybig, Bounds on positive integral solutions of linear diophantine equations, Proceedings of the A.M.S. Vol. 55 Number 2 March 1976, 299-304.

2. I. Borosh, A sharp bound for positive solutions of homogeneous linear diophantine equations, Proceedings of the A.M.S. Vol. 60 October 1976, 19-21. 
3. W. Haken, Theorie der Normal flacken Acta. Math. 105 (1961), 245-375.

4. H. Schubert, Bestimmung der Primfaktorzerlegung von Verkettungen, Math. Zeit., 76 (1961), 116-148.

5. L. B. Treybig, Bounds in piecewise linear topology, Trans. Amer. Math. Soc., 201 (1975), 383-405.

Department of Mathematics

TEXAS A \& M UNIVERSITY

COLlege of SCIENCE

College Station, Texas 77840 